\title{
What Characterizes Safety of Ambient Assisted Living Technologies?
}

\author{
Kerstin DENECKE ${ }^{\mathrm{a}, 1}$ \\ ${ }^{a}$ Bern University of Applied Sciences, Bern, Switzerland
}

\begin{abstract}
Ambient assisted living (AAL) technologies aim at increasing an individual's safety at home by early recognizing risks or events that might otherwise harm the individual. A clear definition of safety in the context of AAL is still missing and facets of safety still have to be shaped. The objective of this paper is to characterize the facets of AAL-related safety, to identify opportunities and challenges of AAL regarding safety and to identify open research issues in this context. Papers reporting aspects of AAL-related safety were selected in a literature search. Out of 395 citations retrieved, 28 studies were included in the current review. Two main facets of safety were identified: user safety and system safety. System safety concerns an AAL system's reliability, correctness and data quality. User safety reflects impact on physical and mental health of an individual. Privacy, data safety and security issues, sensor quality and integration of sensor data, as well as technical failures of sensors and systems are reported challenges. To conclude, there is a research gap regarding methods and metrics for measuring user and system safety in the context of AAL technologies.
\end{abstract}

Keywords. Safety, assistive technologies, AAL, ambient assisted living, security

\section{Introduction}

Assistive technology is "an umbrella term for any device or system that allows individuals to perform tasks they would otherwise be unable to do or increases the ease and safety with which tasks can be performed" [1]. Ambient Assisted Living (AAL) technologies form a subset of assistive technologies. They address safety needs at home such as recognition of frailty and mobility, health and accident prevention, and support in daily living [2]. They also facilitate living at home by smart home elements and they support individuals by monitoring medical parameters, detecting falls, or acting as medical reminders, e.g., reminders on appointments or intake of medicine [3]. In this way, it is assumed that they have potential in enhancing the actual and perceived safety [4], well-being and independence of individuals at home [5]. AAL technologies integrate functionalities that are safe-critical [3]. This creates the demand that AAL systems must be safe, i.e. a user must not suffer from injuries in case system failures occur [6]. Despite the research interest in AAL technologies and the clear objective of AAL to increase safety, it is still unclear what exactly means safety in the AAL context and what shapes this safety. The objective of this study is to synthesize facets of safety in the context of AAL as well as to shape future research endeavors.

\footnotetext{
${ }^{1}$ Corresponding Author, Kerstin Denecke, Bern University of Applied Sciences, Institute for Medical Informatics, Quellgasse 21,2501 Biel, Switzerland; E-mail: kerstin.denecke@bfh.ch.
} 


\section{Methods}

This study follows a qualitative approach using a literature review to identify issues related to safety in the context of AAL. Sub-questions driving this analysis include: 1) Which facets of safety in the AAL context can be distinguished? 2) Which opportunities of AAL for safety are reported? and 3) Which aspects of safety in AAL-related research are described? The search was carried out on June 2 2020. The search strategy covered all articles published during the period 2010-2020. Search terms were.

- PUBMED: ("safety") AND (("self help device") OR ("internet-based intervention") OR ("ambient assisted living")); 45 results

- $\quad$ ACM Digital Library: ("safety") AND (("self help device") OR ("internetbased intervention") OR ("ambient assisted living")); 162 results

- IEEE Xplore: ("safety") AND (("self help device") OR ("internet-based intervention") OR ("Ambient assisted living")); 58 results

- EMBASE: ("safety") AND ("self help device" OR "internet-based intervention" OR "ambient assisted living"); 130 results

Articles were included in this review if: 1) they dealt with AAL technologies or smart home technologies in care contexts, 2) they reported issues related to patient safety and 3) they were published in the following languages: English, German. We excluded papers dealing with social robots, wheelchair technology, social networking systems, rehabilitation technologies or those that did not deal with safety aspects. Duplicates were identified and removed. In order to assess the eligibility of the articles, all titles and abstracts were examined. In a second round, the full-text of the selected articles were extracted and carefully analyzed to confirm their eligibility. The selected articles were included in the qualitative synthesis. Data related to aspects of patient safety, risks and opportunities of AAL systems related to safety and safety assessment methods were extracted from the selected studies. From the results, we derived facets of safety and needs for future research.

Table 1. Types of AAL applications included in the review

\begin{tabular}{ll}
\hline Purpose of the system & System description \\
\hline Emergency detection & Monitoring of activities and risks in the bathroom [7], \\
& Mobile safety alarms [8], \\
& Distinction of safe and unsafe events [9], \\
& Fall detection [10] \\
& Recognition of presence of individuals in rooms [11], \\
Monitoring & Well-being monitoring [12], \\
& Monitoring activities and physical status and suggesting proactive \\
& measures [13] \\
\hline
\end{tabular}

\section{Results}

A total of 395 records was identified, 39 duplicates were removed and 28 papers met the inclusion criteria, and therefore were included in the qualitative synthesis. Seven papers reported on concrete AAL applications [7-13], see Table 1. Eleven papers reported rather on AAL in general or were reviews [2,14-23]. General topics related to safety in AAL systems comprised: ethical issues [23], use, adoption and acceptance [17, 19-22], impact of AAL on quality of life [16], needs and behavior related to AAL [2, 15], AAL design considerations [14] and safety analysis [18]. Ten papers introduced a framework or architecture $[6,10,24-34]$. These frameworks and architectures considered the following 
aspects: Architecture for integration of sensors [10], architecture for complete AAL systems [26, 28], architecture for message transfer in AAL systems [24], safety engineering and certification [6, 25, 29, 30], sensor quality [27], security configuration modelling [34].

\subsection{Facets of Safety}

We can distinguish two facets of safety: system safety and user safety. On the one hand, safety concerns the AAL system as such and its components, i.e. its reliability, correctness, data quality. On the other hand, achieving or increasing user safety is targeted by AAL systems, i.e. physical and mental health of an individual has to be ensured by the application of AAL technologies.

There are different aspects that characterize or impact on system safety. It depends on the data quality (and safety) of its components (e.g. sensors, knowledge base). Errorprone data may result in false positive or false negative events being inferred. This in turn can lead to false alarms, missing alarms and finally leads to patient harm and a loss of safety [27]. Several types of user safety can be distinguished [31]: a) Medical safety realized by monitoring of medical parameters, b) fast safety related to fast assistance in emergencies, (e.g. concerns fall detection or other accidents and their prevention), c) safety of daily living (concerns safety related to technical devices and household aids), d) structuring safety (e.g., realized by reminding on intake of medicine or appointments), e) felt or perceived safety related to the feeling that the person in need of care is not alone.

\subsection{Opportunities}

AAL technologies offer multiple opportunities for patient safety achieved through monitoring and emergency detection. Different aspects are monitored: physiological signals and physical illness (e.g. measurements of pulse, respiration, blood pressure) [22], functional aspects such as general activities, motions, meal intake [22], safety aspects (e.g. detection of fire and gas leaks, automatic light switches, fall detection) [2], social interaction [21,22], and cognitive behavior [22]. Data collected by monitoring technologies can be analyzed or transferred. For example, collected physiological or health-related data can be transferred to a physician for further assessment and consideration [10]. When such data is analyzed, anomalies or risks can be detected and alerts can be generated automatically (e.g. automatic fall detection [33], location detection [8]). If working properly and anomalies are detected, an emergency call or appropriate alerts to caregivers and family members can be sent to prevent serious harm [28]. AAL technologies are reported to reduce fear and insecurity as well as to increase both, genuine and perceived safety [8]. For example, users perceived the localization feature of their assistive technology as most important factor for safety in emergencies. Users felt safe knowing that caregivers/relatives could localize them [8]. AAL applications also include applications for social connectedness (phone calls, communication through videos, virtual participation in groups), and for delivering cognitive or sensory support (reminder for medication, lost key locator), or provide care functions and cognitive support (e.g. medication reminder, verbal assistance for appliances) [2]. They increase health (which is a safety need) or can help in maintaining a certain health status. 


\section{Discussion and Conclusions}

Depending on the AAL system's scope (monitoring or emergency detection), the challenges of safety analysis differ. For monitoring applications, assessing data safety and security might be more relevant. In contrast, for applications aiming at detecting and preventing emergencies, there are large demands regarding reasoning and technical quality. A challenge for run-time system safety analysis of AAL systems is how to learn from hazards without harming individuals. We can imagine a critical incident reporting system as known from hospitals, where critical incidents are reported by the user to let the system learn from those incidents. Such strategy could be even applied by healthy people who use AAL technologies. Their experiences from interaction and alerting could be collected. This could help in improving the systems and increasing the safety. However, it will not help in studying the reliability of systems in detecting safety-critical situations. User safety can only be assessed when the system is implemented in the home of an individual. Metrics to quantify the actual user safety are still missing. A reason might be difficulties in quantifying user safety with its facets medical safety, fast safety etc. Instead, the perceived safety is measured. This is realized by questionnaires and interviews of individuals [32] and of non-professional care providers [31] focusing on usability and acceptance.

I conclude that the existing research in the AAL field addresses safety issues to a very limited extent. Even though AAL technologies are supposed to increase safety, there are no studies available providing proof of efficacy of AAL technologies in increasing safety. First indications exist that the perceived safety increases through the application of AAL technologies [31]. This work demonstrates that there is a need for studies that assess to what extent safety is increased through application of AAL technologies. Furthermore, methods are required for measuring the safety of AAL systems, its components and data transfers. Current literature on AAL technologies consider methods for safety analysis [6] and safety certification [30] only to a very limited extent.

\section{References}

[1] WHO Centre for Health Development. A glossary of terms for community health care and services for older persons, In: WHO Kobe Centre ageing and health technical report; v. 5, Kobe, Japan: WHO Centre for Health Development, WHO/WKC/Tech.Ser./04.2, 2004.

[2] Munteanu C, Salah AA. Multimodal technologies for seniors: challenges and opportunities. In: The Handbook of Multimodal-Multisensor Interfaces: Foundations, User Modeling, and Common Modality Combinations. 2017:1:319-362.

[3] Rashidi P, Mihailidis A. A survey on ambient-assisted living tools for older adults. IEEE journal of biomedical and health informatics. 2012 Dec 31;17(3):579-90.

[4] Offermann-van Heek J, Schomakers EM, Ziefle M. Bare necessities? How the need for care modulates the acceptance of ambient assisted living technologies. International journal of medical informatics. 2019 Jul 1;127:147-56.

[5] Padilla-López JR, Chaaraoui AA, Gu F, Flórez-Revuelta F. Visual privacy by context: proposal and evaluation of a level-based visualisation scheme. Sensors. 2015;15(6):12959-82.

[6] Tiadjio AM, Jamboti K. Requirements and evaluation of safety analysis techniques for ambient assisted living systems. In: 2012 IEEE 23rd International Symposium on Software Reliability Engineering Workshops 2012 Nov 27, p. 319-324.

[7] Zaric $\mathrm{N}$ et al. An example of monitoring system with reasoning module for ambient assisted living applications. In: IEEE EUROCON 2015-International Conference on Computer as a Tool (EUROCON). IEEE, 2015. p. 1-6.

[8] Røhne M, Boysen ES, Ausen D. Wearable and Mobile Technology for Safe and Active Living. In: pHealth 2017, p. 133-139. 
[9] Muñoz A et al. Design and evaluation of an ambient assisted living system based on an argumentative multi-agent system. Personal and Ubiquitous Computing. 2011; 15(4): 377-387.

[10] Kunnappilly A, Marinescu R, Seceleanu C. A model-checking-based framework for analyzing ambient assisted living solutions. Sensors. 2019 Jan;19(22):5057.

[11] Alesii R et al. Short range wireless solutions enabling ambient assisted living to support people affected by the Down syndrome. In: Eurocon 2013. IEEE, 2013. pp. 340-346.

[12] Jing Y et al. An intelligent well-being monitoring system for residents in extra care homes. In: Proc. of the 1st International Conference on Internet of Things and Machine Learning. 2017. pp. 1-6.

[13] Cavallo F, Aquilano M, Arvati M. An ambient assisted living approach in designing domiciliary services combined with innovative technologies for patients with Alzheimer's disease: a case study. American Journal of Alzheimer's Disease \& Other Dementias ${ }^{\circledR}, 2015: 30(1)$, pp. 69-77.

[14] Serbedzija N. Reflective assistance for eldercare environments. In: Proceedings of the 2010 ICSE Workshop on Software Engineering in Health Care. 2010. p. 104-110.

[15] Panek P et al. On the Prototyping of an ICT-Enhanced Toilet System for Assisting Older Persons Living Independently and Safely at Home. In: eHealth. 2017. p. 176-183.

[16] Siegel C, Dorner TE. Information technologies for active and assisted living-Influences to the quality of life of an ageing society. International journal of medical informatics, 2017;100(32):45.

[17] Thoma-Lürken $T$ et al. Facilitating aging in place: A qualitative study of practical problems preventing people with dementia from living at home. Geriatric Nursing. 2018:39(1);29-38.

[18] Mouaffo A, Taibi D, Jamboti K. Controlled experiments comparing fault-tree-based safety analysis techniques. In: Proceedings of the 18th International Conference on Evaluation and Assessment in Software Engineering. 2014. p. 1-10.

[19] Hwang AS et al. Co-designing ambient assisted living (AAL) environments: unravelling the situated context of informal dementia care. BioMed research international, 2015

[20] Klein, B et al. Can telecare contribute to an independent life at home with 100? A glance to the UK and initial experiences of the German LOEWE field test on age appropriate sensor based assistance in real estate. In: 2013 6th Int. Conf. on Human System Interactions (HSI). IEEE, 2013. p. 594-599.

[21] Jaschinski C. Ambient assisted living: towards a model of technology adoption and use among elderly users. In: Proceedings of the 2014 ACM International Joint Conference on Pervasive and Ubiquitous Computing: Adjunct Publication 2014 Sep, p. 319-324.

[22] Al-Jumaily A, Ganesan B, Gowda T, Fong KN, Meena SK, Tong RK. Ambient assisted living technologies for older adults with cognitive and physical impairments: a review. European Review for Medical and Pharmacological Sciences. 2019: 23(23):10470 - 10481.

[23] Novitzky P, Smeaton AF, Chen C, Irving K, Jacquemard T, O’Brolcháin F, O'Mathúna D, Gordijn B. A review of contemporary work on the ethics of ambient assisted living technologies for people with dementia. Science and engineering ethics. 2015;21(3):707-65.

[24] Hossain MA, Parra J, Alamri A. Safety-enabled restful messaging in Ambient-Assisted Living. In: 2015 IEEE International Conference on Multimedia \& Expo Workshops (ICMEW). IEEE, 2015. p. 1-6.

[25] Schneider D, Trapp M. Runtime safety models in open systems of systems. In: 2009 Eighth IEEE International Conference on Dependable, Autonomic and Secure Computing. IEEE, 2009. p. 455-460.

[26] Nehmer J et al. Living assistance systems: an ambient intelligence approach. In: Proceedings of the 28th international conference on Software engineering. 2006. p. 43-50.

[27] McNaull J, Augusto JC, Mulvenna M, McCullagh P. Data and information quality issues in ambient assisted living systems. Journal of Data and Information Quality (JDIQ). 2012;4(1):1-5.

[28] De Paola A, Ferraro P, Gaglio S, Re GL, Morana M, Ortolani M, Peri D. An ambient intelligence system for assisted living. AEIT International Annual Conference 2017, p. 1-6.

[29] Schneider D, Becker M, Trapp M. Approaching runtime trust assurance in open adaptive systems. In: 6th Intern. Symp. on Software Engineering for Adaptive and Self-Managing Systems. 2011. p. 196-201.

[30] Schneider D, Trapp M. Conditional safety certification of open adaptive systems. ACM Transactions on Autonomous and Adaptive Systems (TAAS). 2013;8(2):1-20.

[31] Offermann-van Heek J, Ziefle M. Nothing else matters! Trade-offs between perceived benefits and barriers of AAL technology usage. Frontiers in public health. 2019;7:134.

[32] Queiros A, Dias A, Silva AG, Rocha NP. Ambient assisted living and health-related outcomes-a systematic literature review. Informatics. 2017; 4(3):19.

[33] Dupuy L, Froger C, Consel C, Sauzéon H. Everyday functioning benefits from an assisted living platform amongst frail older adults and their caregivers. Frontiers in aging neuroscience. 2017 Sep 28;9:302.

[34] Hoque E et al. Trust based security auto-configuration for smart assisted living environments. In: Proceedings of the 2nd ACM workshop on Assurable and usable security configuration. 2009. S. 7-12. 